\title{
Empleo manufacturero de los estados del centro de México. Análisis shift- share tradicional y con modificación de estructuras, 1998-2018
}

\section{Liliana Rendón Rojas, Pablo Mejía Reyes,} Miguel Ángel díaz Carreño*

\section{RESUMEN}

El objetivo de este trabajo es analizar la dinámica del empleo de los 21 subsectores de la manufactura de los estados del centro de México, a través de la técnica shitf-share tradicional y con modificación de estructuras, complementada con el cociente de localización para medir la especialización productiva. El estudio se inscribe en el periodo 19982018, lo que permite contrastar la evidencia previa y posterior a la Gran Recesión, evento que generó profundos ajustes sectoriales y regionales en la actividad productiva nacional y regional. Se encuentra que la especialización productiva juega un papel importante en el resultado de estados "ganadores" y "perdedores" de empleo. Con ambas técnicas se observan consecuencias parecidas: destrucción de fuentes de empleo en la mayoría de las entidades, principalmente en la Ciudad de México, donde el subsector más afectado fue el 336. Fabricación de equipo de transporte. A lo largo de dos décadas, el estado con mejor desempeño es Guanajuato.

Palabras claves: Economía regional, Shift-share, Empleo manufacturero, Centro de México.

Clasificación JEL: O14, O18, R11; R19

* Profesores-investigadores del Centro de Investigación en Ciencias Económicas de la Facultad de Economía de la Universidad Autónoma del Estado de México, México.

Correo-e: lrendonr@uaemex.mx; pmejiare@uaemex.mx y madiazc@uaemex.mx, respectivamente. 


\section{ABSTRACT}

Manufacturing employment in the central Mexican states. Traditional shift-share analysis and with modification of structures, 1998-2018

The aim of this paper is to analyze the employment dynamics of the 21 manufacturing subsectors of the states of Central Mexico, through the traditional shitf-share technique and with modification of structures, complemented by the localization coefficient to measure productive specialization. The study is part of the period 1998-2018, which allows to contrast the evidence before and after the Great Recession, an event that generated deep sectoral and regional adjustments in national and regional productive activity. Productive specialization is found to play an important role in the outcome of "winners" and "losers" employment states. Both techniques lead to similar consequences: destruction of sources of employment in most states, mainly in Mexico City, where the most affected subsector was 336 Manufacturing transport equipment. Over the course of two decades, the best performing state is Guanajuato.

Keywords: regional economy, shift-share, manufacturing employment, central Mexico.

JEL classification: O14; O18; R11; R19.

\section{INTRODUCCIÓN}

Durante las últimas cuatro décadas, el crecimiento económico y la generación de empleos en México no han alcanzado los niveles esperados; este periodo coincide con el tránsito de un modelo de desarrollo de economía esencialmente cerrada y dirigida por el Estado a otro de economía abierta y de mercado. En este sentido y debido a una serie de factores asociados a las limitantes del modelo de desarrollo, así como por un conjunto de choques externos adversos y errores u omisiones de política económica, la producción redujo su tasa de crecimiento promedio de $6.5 \%$, durante el periodo $1940-1981$, a $2.2 \%$, en $1982-2010 .^{1}$

Cárdenas (1996), Moreno-Brid y Ros (2010) y Mejía y Torres (2014) analizan las reformas estructurales del modelo de desarrollo. Véase también Ros (2014 y 2015) para una discusión sobre los factores que explican el menor ritmo de crecimiento de la economía mexicana durante las últimas décadas. 
A partir de entonces, la liberalización del comercio y de la inversión extranjera, la desregulación de los mercados y la reprivatización de las empresas públicas, entre otras reformas, transformaron la estructura y la dinámica de la economía mexicana. En particular, el sector manufacturero ganó un lugar preponderante después de que emprendió una profunda reestructuración productiva para reorientar su producción hacia el exterior. El aumento de los flujos de capital internacional y la disponibilidad de factores productivos en la cantidad y calidad suficientes en el país hicieron posible el ajuste, por lo que este sector pronto alcanzó una fuerte integración a los flujos internacionales de capital y comercio, con lo que logró captar una importante proporción de inversión extranjera y vender volúmenes crecientes de mercancías al exterior (Fuentes, 2003; Dávila, 2004; Erquizio y Ramírez, 2017).

A pesar de que el proceso de terciarización de la economía mexicana se ha profundizado y la manufactura ha reducido su participación en el Producto Interno Bruto (PIB) nacional, para ubicarse en torno a 17.0 puntos porcentuales entre 1999 y 2018, las cualidades que suelen atribuirse a este sector-capacidad de innovación, generación de economías externas y de escala, y contratación de mano de obra calificada, entre otras- han favorecido su reestructuración y su inserción a la economía internacional, principalmente a la de Estados Unidos de América. ${ }^{2}$ En efecto, durante el periodo de estudio, la manufactura ha contribuido con más de $80 \%$ a las exportaciones totales y ha captado poco menos de $50 \%$ en promedio de la inversión extranjera directa (IED) total, ${ }^{3}$ lo que ha provocado una elevada sincronización de los ciclos no sólo de la manufactura sino de las dos economías (Mejía y Campos, 2011; Mejía, 2011).

En particular, a raíz de esa significativa integración internacional, México ha enfrentado dos choques externos que han afectado profundamente su estructura productiva. El primero se relaciona con el ingreso de China a la Organización Mundial de Comercio (OMC) en 2001, que significó no sólo su eventual desplazamiento del mercado esta-

2 El crecimiento de la IED y de las exportaciones han llevado a una fuerte integración de la economía nacional a la de Estados Unidos, su principal socio comercial, reflejada en la alta sincronización de los ciclos, no sólo de la manufactura, sino de las dos economías.Véanse Mejía y Campos (2011) y Mejía (2011).

3 Las cifras se obtuvieron de la página oficial del Instituto Nacional de Estadística y Geografía de México (www.inegi.org.mx). 
dounidense como uno de sus principales proveedores, sino también la sustitución de producción nacional por importaciones chinas, especialmente de insumos (Iacovone et al., 2013; Mendez, 2015). El segundo se refiere a la Gran Recesión, originada en EUA y transmitida al resto del mundo a través de sus vínculos financieros y comerciales, que implicó una caída acumulada en la manufactura mexicana igual a $17.5 \%$ entre julio de 2007 y mayo de 2009, la más grande durante las recesiones registradas desde 1980 (Mejía, 2020).

Históricamente, la región centro de México ha ocupado un lugar preponderante en la política y la economía nacionales. No obstante, durante las últimas décadas se ha desarrollado un proceso de desconcentración de la vida económica y social del país que ha conducido a una disminución paulatina de su peso en los indicadores económicos más importantes, incluso en fechas recientes. ${ }^{4}$ Por ejemplo, su participación en el empleo nacional ha disminuido con los años, ya que en 1998 aportaba al empleo manufacturero total un promedio de $40 \%$, cifra que disminuyó a 38 y a 34\%, en 2008 y 2018, respectivamente. Sin embargo, algunos autores, como Issac-Egurrola y Quintana (2012); Cañedo et al. (2012); Mendoza (2012); Ramírez (2012) y Rodríguez (2010), coinciden en señalar que a pesar de la relocalización de la actividad económica hacia los estados del norte y la terciarización de la economía, los estados del centro de México aún mantienen su primacía y liderazgo en términos de mercado interno.

A pesar de la evidente importancia de esta región, existen relativamente pocos estudios que analicen la dinámica del empleo de los estados del centro en su conjunto, que incluye al Estado de México, Puebla, Tlaxcala, Morelos, Hidalgo, Distrito Federal, Querétaro y Guanajuato. ${ }^{5}$ En este contexto, el objetivo del presente documento es analizar la dinámica del empleo manufacturero de los estados del centro de México mediante la técnica shift-share tradicional y una versión de modificación de estructuras. Específicamente se contrastan las características del empleo manufacturero de sus 21 subsectores en los periodos 1998-2008 y 2008-2018 a partir de información censal. El periodo de estudio se inscribe en la fase de apertura comercial de la economía mexicana, al

4 Véanse los análisis de relocalización de la actividad productiva de Fuentes (2003) y Dávila (2004), y el estudio de su estado actual en Banco de México (2018).

5 Aquí se considera la regionalización que utiliza el Banco de México (2018). 
tiempo que permite contrastar la evidencia previa y posterior al ingreso de China a la Organización Mundial de Comercio (2001) y a la Gran Recesión, eventos que, sin duda, generaron profundos ajustes sectoriales y regionales en la actividad productiva nacional.

El documento se divide en tres secciones adicionales. En la primera se lleva a cabo una revisión de la literatura; en la segunda se presentan la metodología shift-share en su versión tradicional y con modificación de estructuras, así como el cálculo de la especialización productiva; en la tercera se discuten los resultados y, por último, se establecen las conclusiones.

\section{BREVE REVISIÓN DE LA LITERATURA}

La técnica shift-share, aparecida a principios de la década de 1940 (Houston, 1967), tiene como objetivo principal la medición de cambios (geográficos) en algunos agregados macroeconómicos; no obstante, una de las principales aplicaciones de este enfoque se ha dedicado al análisis del crecimiento económico regional. A partir de la versión original del modelo shift-share se han propuesto una gran diversidad de ajustes al mismo, como versiones dinámicas y probabilísticas, hasta la incorporación de efectos espaciales.

Al respecto, Boisier (1980) y Lira y Quiroga (2009) aseveran que la técnica shift-share establece que el crecimiento de algún agregado económico es mayor en algunos sectores o regiones que en otros.

Teniendo en cuenta que el enfoque estático shift-share no considera los cambios continuos tanto a nivel estructural como sobre el tamaño del empleo total de la región en el periodo de estudio, Barff y Knight (1998) calcularon el efecto del crecimiento nacional, el efecto estructural y el efecto competitivo sobre una base anual, para posteriormente sumar los resultados en el periodo de estudio y obtener una medida más precisa de los cambios en el empleo entre los tres efectos shift-share. Este enfoque, denominado análisis shift-share dinámico, también permite identificar años inusuales, así como años de transición económica.

En esta misma variante están los siguientes artículos. Por ejemplo, Herath (2013) analizó el crecimiento del empleo y las implicaciones de política para el desarrollo económico de Virginia del Oeste. El estudio utiliza datos de empleo de 1970 a 2007; los resultados muestran que la 
agricultura, minería y manufactura han perdido la relevancia que tenían en la economía de Virginia del Oeste.

Por su parte, Sirakaya et al. (2002) examinan el funcionamiento de la industria del turismo con datos de series de empleo para el estado de Texas y el total de EUA. Los hallazgos muestran que, en comparación con la media de este país norteamericano, el cambio en el empleo en Texas se debió principalmente a la fortaleza de la economía nacional y no a la competitividad de la región o la estructura sectorial.

Asimismo, Bonet (1999) presenta un análisis del crecimiento regional en Colombia. Éste permite identificar si la especialización productiva de ciertas regiones ha sido la causa de su avance o retroceso en el contexto nacional. Aquellas regiones que han concentrado su estructura productiva en sectores poco dinámicos o, por el contrario, las que se han concentrado en renglones dinámicos, presentarán un comportamiento muy diferente al observado en el promedio nacional. De acuerdo con los resultados de este estudio, se plantea que el uso de un modelo dinámico shift-share permite eliminar un problema teórico inherente en el método clásico estático.

En la segunda mitad de los años 70 se reconoció la necesidad de incorporar la interacción espacial en dicho enfoque, particularmente en el trabajo de Hewings (1976). A partir de entonces se ha desarrollado una enorme literatura en torno a la aplicación de la técnica shift-share con efectos espaciales. Enseguida se citan algunos de estos trabajos.

Primeramente, Hanham y Banasick (2000) examinaron el papel de la estructura espacial en los cambios del empleo manufacturero regional en Japón, en contraste con los estudios tradicionales que se centran en el papel de la estructura industrial. En general, los resultados muestran que hubo un progresivo subdesarrollo en el centro de las regiones asociado a la caída del producto y de la productividad. Las regiones periféricas del país se caracterizaron por un desarrollo vinculado al aumento de la producción y la productividad.

Por su lado, Nazara y Hewings (2004) incorporaron la estructura espacial dentro del análisis shift-share. De esta manera, al análisis de descomposición tradicional se agregó la interacción interregional. Por lo tanto, este trabajo desarrolló una taxonomía de la descomposición de la tasa de crecimiento regional.

A su vez, Mayor y López (2009) analizaron la influencia de efectos espaciales en la evolución del empleo regional en España a partir de dos 
técnicas no paramétricas propuestas: un análisis espacial shift-share y un filtro espacial, con lo que se mejoró la explicación de las diferencias existentes.

Con base en la metodología anteriormente citada, el trabajo de Rendón et al. (2019a) analiza el empleo de los 21 subsectores de la manufacturera de los municipios de las zonas metropolitanas del Valle de México y Toluca. Estos autores concluyen que los subsectores que benefician a sus vecinos son los de baja tecnología.

Debido al gran auge que ha experimentado la aplicación del modelo shift-share, con efectos espaciales a la versión clásica, en la actualidad continúa utilizándose -con algunos ajustes- de forma masiva. En seguida se describen algunos casos.

Knudsen y Barff (1991) muestran que el análisis convencional shiftshare y el estocástico generan conclusiones similares. Varias de las críticas de la técnica de los modelos estocásticos son dirigidas debido a que se permite la realización de pruebas de hipótesis y al mismo tiempo preserva la viabilidad del enfoque convencional. De esta manera, se sugiere que el análisis estocástico shift-share puede ser empleado sin ninguna restricción.

Posteriormente, Knudsen (2000) demuestra la relevancia de dos formas probabilísticas de modelos shift-share. Éstos, según este autor, dan una mayor ventaja sobre la metodología clásica de dicho enfoque, debido a que permiten realizar pruebas de hipótesis acerca de los cambios en el nivel de empleo o valor agregado por la región, o sector productivo.

Por su parte, Márquez et al. (2009) proponen una forma alternativa de incorporar la dimensión sectorial dentro de los componentes del crecimiento regional dado en el análisis shift-share tradicional. La nueva metodología detalla la forma en que la dinámica de un sector específico en una región es influenciada por el desempeño de otros sectores, éste último dividido en efectos nacionales, estructurales y diferenciales. Los resultados revelan cómo estos nuevos componentes pueden dar nuevas ideas en el análisis de procesos de crecimiento económico sectorial y regional.

A su vez, Esteban (2000) analiza el grado de desigualdad interregional en la productividad agregada por trabajador en la Unión Europea con la técnica shift-share. Se establece que dicha desigualdad puede ser atribuida a diferencias en la composición sectorial de las actividades 
más que a brechas en la productividad, que son uniformes a través de los sectores. Se demuestra que la especialización regional tiene un papel menor y que las diferencias interregionales pueden ser principalmente explicadas sólo por brechas uniformes en productividad.

Por su lado, Fotopoulos et al. (2009) analizan los efectos del comercio internacional, la especialización relativa y la estructura industrial regional en el crecimiento local del empleo en Grecia durante 1995-2003. Los resultados obtenidos resaltan la importancia de las condiciones locales, las desviaciones regionales del ciclo económico y las tendencias sectoriales. A su vez, la especialización regional tiene, en promedio, un efecto positivo en el crecimiento del empleo regional; mientras que,la dirección de los efectos de las industrias (industry-mix) depende de la especialización en los sectores con crecimiento o en declive. Aunado a ello, las importaciones tienen efectos negativos en el empleo; el efecto positivo de las exportaciones es débil y la demanda doméstica se mantiene como un factor positivo importante.

Finalmente, Barrios (2008) lleva a cabo el cálculo de la influencia de la especialización productiva en el comportamiento de las exportaciones colombianas con la metodología shift-share tradicional y con modificación de estructuras durante el siglo XIX y XX, y encuentra tres periodos importantes que explican las exportaciones de Colombia dada su especialización productiva.

\section{Metodología}

El shift-share (cambio y participación) es una técnica de análisis del crecimiento de la producción y del empleo de las regiones, propuesta por Dunn (1960), que tiene como objetivo cuantificar los cambios o sesgos geográficos en la actividad económica. En este artículo se hace uso de la técnica shift-share tradicional y con modificación de estructuras para estudiar la dinámica del empleo manufacturero de los ocho estados del centro de México, y se identifica su especialización productiva. La información estadística se obtuvo de los censos económicos del INEGI que registran al personal ocupado del sector para los años 1998, 2008 y 2019. 


\section{a) Análisis shift-share tradicional}

La técnica shift-share se basa en la descomposición del crecimiento de una variable en dos componentes: el efecto estructural y el efecto diferencial. El primero refleja el efecto que el crecimiento de una economía tiene sobre otra, mientras que el segundo muestra el impacto de una determinada especialización sectorial sobre el crecimiento de la variable en estudio. Por lo tanto, este análisis permite identificar si la especialización productiva de ciertas regiones ha sido la causa de su avance o retroceso en el contexto nacional. Aquellas regiones que han concentrado su aparato productivo en sectores poco dinámicos o, por el contrario, las que se han concentrado en sectores dinámicos, presentarán un comportamiento muy diferente al observado en el promedio nacional (Bonet, 1999).

Basados en Lira y Quiroga (2009), así como en Torres et al. (2009), se distinguen sus componentes en los efectos estructural y diferencial. El primero, también denominado efecto proporcional y designado por $E E_{j}$, se define por sectores de la variable en el espacio geográfico considerado con base en la estructura interna; refleja la diferencia de dinámica entre la región y el país, derivada de una "estructura intersectorial distinta" entre ambos. Esto resulta de las diferencias de crecimiento de los diversos sectores en el ámbito nacional, combinado con el peso relativo de éstos en los ámbitos nacional y regional. Es decir, el efecto estructural indica si existe especialización del empleo en algún subsector de rápido crecimiento (resultado positivo) o no (resultado negativo). De acuerdo con Boisier (1980), la importancia relativa de los subsectores económicos en una región (su estructura económica) constituye uno de los elementos determinantes de las posibilidades de crecimiento regional en un periodo dado (Ecuación 1).

Formalmente,

$$
E E_{j}=\sum_{i} V_{i j}(0) * \sum_{i}\left\{r S_{i} *\left[\frac{V_{i j}(0)}{\sum_{i} V_{i j}(0)}-\frac{\sum_{j} V_{i j}(0)}{\sum_{i} \sum_{j} V_{i j}(0)}\right]\right\}
$$

Esta expresión puede ser descompuesta en:

$$
E E_{j}=\sum_{i}\left\{V_{i j}(0) *\left[\frac{\sum_{j} V_{i j}(t)}{\sum_{j} V_{i j}(0)}-\frac{\sum_{i} \Sigma_{j} V_{i j}(t)}{\sum_{i} \Sigma_{j} V_{i j}(0)}\right]\right\}
$$


donde $i$ denota el $i$-ésimo sector (o rama de actividad) y $j$ a la $j$-ésima región (o entidad geográfica en general); $V$ se refiere a la variable de análisis y 0 y $t$ son el año base y el año final. Además, se define el cociente de variación del sector $i$ entre 0 y $t$ como:

$$
r S_{i}=\sum_{j} V_{i j}(t) / \sum_{j} V_{i j}(0)
$$

y el cociente de variación del patrón de comparación entre 0 y t como:

$$
r S R=\sum_{i} \sum_{j} V_{i j}(t) / \sum_{i} \sum_{j} V_{i j}(0)
$$

A partir de los valores de $E E_{j}$ se tienen tres posibles casos: a) $E E_{j}>0$ indica que la estructura del empleo o producción regional es más favorable para el crecimiento que la estructura nacional; b) $E E_{j}=0$ implica que la estructura del empleo o producción regional es igual a la estructura del empleo nacional, y c) $E E_{j}<0$ sugiere que la estructura del empleo o producción regional es menos favorable para el crecimiento que la estructura nacional.

Por otro lado, el efecto diferencial (Ecuación 3) mide el grado de heterogeneidad, es decir, el ritmo diferente de crecimiento de los sectores económicos por separado en cada espacio geográfico; se representa por $E D_{j}$ Recoge la dinámica de cada sector i en la región j comparada con la dinámica del mismo sector en el patrón de comparación, se expresa:

$$
E D_{j}=\sum_{i}\left[V_{i j}(t)-V_{i j}(0) * r S_{i}\right]
$$

Equivalentemente:

$$
E D_{j}=\sum_{i}\left\{V_{i j}(t)-V_{i j}(0) *\left[\frac{\sum_{j} V_{i j}(t)}{\sum_{j} V_{i j}(0)}\right]\right\}
$$

En este caso, se tienen las siguientes posibilidades: a) $E D_{j}>0$ indica que la región contiene sectores con ritmo de crecimiento superiores a los nacionales; b) $E D_{j}=0$ corresponde al caso en el que los ritmos de crecimiento regional son iguales a los del país, y c) $E D_{j}<0$ implica que la región tiene ritmos de crecimiento inferiores a los del país. 
Este efecto muestra que si una región presenta mayor competitividad en relación con el resto de las regiones en el empleo de un determinado subsector, existe, por tanto, una dinámica positiva cuando se compara con el promedio nacional (estatal) del subsector, dado que ciertas regiones presentan condiciones más favorables que otras para la expansión de algunas actividades. Las regiones con efectos diferenciales positivos son las que gozan de condiciones de accesibilidad a los mercados o a los recursos productivos en comparación con otras.

El efecto total $E T_{j}$ (Ecuación 5) es la suma algebraica de ambos efectos; compara lo que ocurrió en la región en el año $t$ con lo que habría ocurrido si la región se hubiera comportado como el patrón de comparación en el periodo de análisis. Por lo tanto, muestra una dinámica relativa al comparar el valor final (en el año $t$ ) de la variable en la región $j$ con el valor que hipotéticamente habría tenido esta variable si la región, en términos de crecimiento, se hubiera comportado como el país o el patrón de comparación elegido. El valor "esperado o hipotético" se obtiene al aplicar el cociente de variación global (rSR) al valor inicial de la variable (en el año 0). Es decir:

$$
E T_{j}=\sum_{i} V_{i j}(t)-\sum_{i} V_{i j}(0) * r S R
$$

Equivalentemente:

$$
\begin{aligned}
& E T_{j}=\sum_{i} V_{i j}(t)-\sum_{i} V_{i j}(0) *\left[\frac{\sum_{i} \sum_{j} V_{i j}(t)}{\sum_{i} \sum_{j} V_{i j}(0)}\right] \\
& E T_{j}=E D_{j}+E E_{j}
\end{aligned}
$$

Un efecto total positivo indica un crecimiento relativo mayor de la variable regional que el crecimiento relativo de la misma variable a escala nacional, o lo que es lo mismo: una mayor dinámica regional que nacional.

Por otro lado, de acuerdo con Boisier (1980), al analizar la combinación de los efectos estructurales y diferenciales es posible clasificar a 
los estados en seis tipos, que da como resultado "ganadores" o "perdedores"6 (Cuadro 1).

\section{CUADRO 1}

TIPOLOGÍA DE RESULTADOS DEL MÉTODO

\begin{tabular}{|c|c|c|c|}
\hline \multicolumn{2}{|c|}{ Efecto total $>0$} & \multicolumn{2}{|c|}{ Efecto total $<0$} \\
\hline Tipo I & $\begin{array}{c}\text { ED + } \\
\text { Tipo II A }\end{array}$ & Tipo IV & $\begin{array}{l}\text { ED - } \\
\text { ED - }\end{array}$ \\
\hline Tipo II A & $\begin{array}{c}\mathrm{ED}+ \\
\mathrm{EE}+ \\
\text { Si IEDI < IEEI }\end{array}$ & Tipo II B & $\begin{array}{c}E D- \\
E E+ \\
\text { Si IEDI > IEEI }\end{array}$ \\
\hline Tipo III A & $\begin{array}{c}E D+ \\
E E- \\
\text { Si IEDI > IEEI }\end{array}$ & Tipo III B & $\begin{array}{c}\text { ED + } \\
\text { EE - } \\
\text { Si IEDI < IEEI }\end{array}$ \\
\hline
\end{tabular}

Fuente: elaborado con datos de Boisier (1980) y Mitchell et al. (2005).

\section{b) Análisis shift-share con modificación de estructuras}

El análisis shift-share con modificación de estructuras surge de la necesidad de involucrar un componente dinámico en su desarrollo con el fin de conocer cuál ha sido la evolución de la variable. Stilwell (1969) formuló el shift-share dinámico modificado, donde el efecto estructural $(E E)$ se calcula tal como se hace en el análisis tradicional, pero se introduce un nuevo efecto denominado estructural inverso $(E I)$, que mide el cambio que se habría producido dados: (a) el cociente de variación de cada sector en el nivel nacional durante el periodo de estudio, y (b) la estructura de cada región al final del periodo. Representa el cambio que se habría esperado, teniendo en consideración la estructura regional al final del periodo. En términos dinámicos, se hablaría de los efectos resultantes de las diferencias en la estructura productiva entre el periodo inicial y final.

La aportación del análisis shift-share modificado es que la comparación entre $E I$ y $E E$ sirve para cuantificar la importancia del cambio estructural. A éste se le llama efecto estructural modificado (EM) o efecto reasignación, ya que sirve para indicar si la especialización regional ha evolucionado hacia sectores con un mayor dinamismo (caso

6 Lira y Quiroga (2003) y Silva (2003) denominan “potencialmente ganadora” a las regiones que han crecido por sobre la media nacional o "potencialmente perdedora" en caso contrario. 
en el que el $E M$ es positivo) o si, por el contrario, el cambio estructural se caracteriza por una especialización creciente en sectores en retroceso (caso en el cual el efecto $E M$ es negativo). Con este nuevo efecto no sólo se puede indagar si la región posee componentes estructurales importantes, sino que también se podrá saber cuál es la tendencia en el mediano y largo plazo de su estructura productiva. En otras palabras, permite identificar si la región se está reorientando hacia sectores más o menos productivos. Con ello se podrá tener una aproximación sobre las tendencias que se podrían esperar en un mediano plazo (Lira y Quiroga, 2009).

El Efecto Estructural Inverso $\left(E I_{j}\right)$ se define como:

$$
E I_{j}=\sum_{i}\left\{V_{i j}(t) *\left[\frac{\sum_{i} \sum_{j} V_{i j}(0)}{\sum_{i} \sum_{j} V_{i j}(t)}-\frac{\sum_{j} V_{i j}(0)}{\sum_{j} V_{i j}(t)}\right]\right\}
$$

El Efecto Estructural Modificado $\left(E M_{j}\right)$ es el resultado de:

$$
E M_{j}=E I-E E
$$

La diferencia entre el efecto estructural inverso y el efecto estructural indica el cambio neto en la estructura de la región en los dos periodos, denominado efecto estructural modificado.

Si se resta el efecto estructural modificado al efecto diferencial, se obtiene el efecto regional modificado $\left(E R M_{j}\right)$ o efecto diferencial residual, que también es igual al efecto total menos el efecto estructural y el efecto estructural modificado, como se aprecia en la siguiente expresión:

$$
E R M_{j}=E T_{j}-E E_{j}-E M_{j}=E T_{j}-E I_{j}
$$

Este efecto captura tanto los comportamientos regionales y nacionales como los que resultan de los cambios en la estructura productiva al final del periodo. Por ello, es una variación observada que se atribuye a factores de la región.

Al respecto, Stilwell (1969) establece una tipología al combinar el resultado de los efectos de cada región, como se muestra en el Cuadro 2. Argumenta que esta clasificación es más útil que la del shift and share tradicional, porque ayuda a identificar áreas que aún no sufren de la 
disminución de empleo, pero que es probable que lo hagan en el futuro. También se pueden identificar las regiones que tienen cambios netos en el pasado y cómo se podrían revertir en el futuro.

CUADRO 2

TIPOLOGÍA DE RESULTADOS CON ANÁLISIS DIFERENCIAL-ESTRUCTURAL MODIFICADO

\begin{tabular}{|c|c|c|c|c|}
\hline Tipo & $\mathrm{EEj}$ & EEMj & ERMj & Condición \\
\hline 1 & + & + & + & \\
\hline 2 & - & - & - & \\
\hline 3 & + & + & - & Si I EEj + EEMj I > I ERMj I \\
\hline 4 & + & + & - & Si I EEj + EEMj I < I ERMj I \\
\hline 5 & + & - & + & Si I EEj + EEMj I > I ERMj I \\
\hline 6 & + & - & + & Si I EEj + EEMj I < I ERMj I \\
\hline 7 & - & + & + & Si I EEMj + EEMj I > I EEj I \\
\hline 8 & - & + & + & Si I EEMj + EEMj I < I EEj I \\
\hline 9 & + & - & - & Si I EEj I > IEEMj + ERMj I \\
\hline 10 & + & - & - & Si I EEj I < IEEMj + ERMj I \\
\hline 11 & - & + & - & Si I EEMj I > EEj + ERMj I \\
\hline 12 & - & + & - & Si I EEMj I < EEj + ERMj I \\
\hline 13 & - & - & + & Si I EEMj I > EEj + EEMj I \\
\hline 14 & - & - & + & Si I EEMj I <EEj + EEMj I \\
\hline
\end{tabular}

Fuente: elaborado con datos de Lira y Quiroga (2009) y Stilwell (1969).

Así como en la tipología del shift-share tradicional, para el modificado también existen tipos de regiones. Las regiones con efecto regional modificado positivo son "ganadoras", condición más pronunciada en las regiones tipo 1. Son "perdedoras" las de efecto negativo, condición más pronunciada en las regiones tipo 2, y así sucesivamente (Lira y Quiroga, 2009). 


\section{RESUltados}

En este análisis shift-share para los años 1998-2008 y 2008-2018 se comparó el desempeño del personal ocupado de los 21 subsectores de la industria manufacturera ${ }^{7}$ de los ocho estados del centro de México con el desempeño que habrían tenido si se hubieran comportado como el total en ese mismo periodo. ${ }^{8}$

\section{a) Shift-share tradicional}

Dado que esta técnica parte de que el crecimiento es mayor en algunos sectores y regiones, se observa que existen grandes diferencias en los componentes del shift-share para los estados de México. En el Cuadro 3 se presentan los resultados de los efectos, clasificados conforme al Cuadro 1, lo que permite obtener los tipos de regiones para los dos periodos de estudio.

Cabe mencionar que también se obtuvo el cociente de localización de cada uno de los estados estudiados para determinar los subsectores en los que se especializan en los diferentes años. Al respecto, algunos autores, como Mendoza (2003) y Fuentes (2003), sugieren que la especialización industrial promueve el crecimiento a través de la generación de economías de escala internas y externas, en tanto que Ocegueda et al. (2009) muestran que hay una relación positiva entre el crecimiento y la especialización cuando ésta se orienta a sectores clave. En el Cuadro $1 \mathrm{del}$ anexo se presenta este cociente para los periodos 1998-2008 y 2008-2018. Como es bien sabido, si el cociente es mayor a uno, la acti-

7313 Fabricación de insumos textiles y acabado de textiles; 314 Fabricación de productos textiles, excepto prendas de vestir; 315 Fabricación de prendas de vestir; 316 Curtido y acabado de cuero y piel, y fabricación de productos de cuero, piel y materiales sucedáneos; 321 Industria de la madera; 322 Industria del papel; 323 Impresión e industrias conexas; 324 Fabricación de productos derivados del petróleo y del carbón; 325 Industria química; 326 Industria del plástico y del hule; 327 Fabricación de productos a base de minerales no metálicos; 331 Industrias metálicas básicas; 332 Fabricación de productos metálicos; 333 Fabricación de maquinaria y equipo; 334 Fabricación de equipo de computación, comunicación, medición y de otros equipos, componentes y accesorios electrónicos; 335 Fabricación de accesorios, aparatos eléctricos y equipo de generación de energía eléctrica; 336 Fabricación de equipo de transporte; 337 Fabricación de muebles, colchones y persianas, y 339 Otras industrias manufactureras.

8 En este trabajo se considera al "sector" como el subsector manufacturero; la "región" se refiere al estado y el "nacional" a la región centro en su conjunto. 
vidad analizada tiene mayor relevancia en el orden regional, por lo que el estado se especializa en ese subsector. ${ }^{9}$

\section{CUADRO 3}

ANÁLISIS SHIFT-SHARE (ETJ, EDJ Y EEJ)

PeRsonal ocupado de los ESTADOS del CENTRO de MÉxico. $1998-2008$ Y 2008-2018

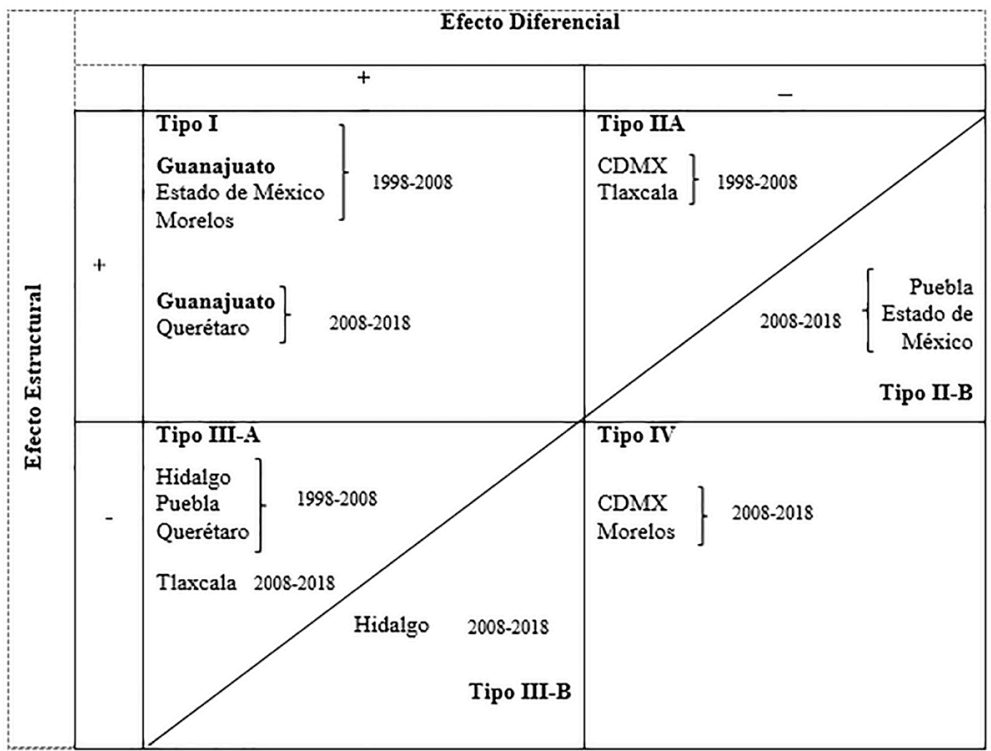

Fuente: elaborado con datos del INEGI (1999, 2009 y 2020).

De acuerdo con los efectos identificados, se observa que el efecto estructural es negativo en los estados de Hidalgo, Puebla, Querétaro (1998-2008), Tlaxcala, Hidalgo, Ciudad de México y Morelos (20082018), lo que revela una menor tasa de crecimiento del empleo que la región centro en su conjunto. Esta situación se explica por el menor dinamismo de sus principales sectores en los que se especializa, de

9 En este trabajo se calculó la especialización interregional, según la cual una región está especializada en los sectores en los que se tiene un tamaño relativo mayor que en el estado o país. Qij representa el cociente de localización. $Q_{i j}=\frac{V_{i j}}{\sum i v_{i j}}: \frac{\sum j V_{i j}}{\sum i \sum j V_{i j}}$, donde $V_{i j}$ es el valor de $V$ (variable de estudio) correspondiente al sector " $i$ " de la rēgiỏn "j"; $\sum i \quad V_{i j}$ denota el valor de $V$ correspondiente al total regional; $\sum_{j} V_{i j}$ es el valor de $V$ correspondiente al total sectorial, y $\sum_{i} \sum_{j} V_{i j}$ corresponde al valor de $V$ a nivel nacional (Boisier, 1980; Blair, 1995). 
acuerdo con el valor del cociente de localización (madera, industria alimentaria, bebidas y tabaco, insumos y productos textiles, prendas de vestir) y por presentar una estructura productiva con escasa presencia de sectores dinámicos en el nivel regional.

A su vez, el resultado es positivo en Guanajuato, Estado de México, Morelos, Ciudad de México y Tlaxcala (1998-2008), así como Querétaro, Guanajuato, Estado de México y Puebla (2018-2018), lo que refleja una especialización estatal en sectores de rápido crecimiento (química, maquinaria y equipo, plástico y hule, productos metálicos, equipo de transporte y aparatos eléctricos) a principios de cada periodo.

Respecto al efecto diferencial, cada uno de los subsectores de un estado se comporta de forma diferente en otros estados. En el periodo 1998-2008 existía un bajo dinamismo en los 21 subsectores de la manufactura en la Ciudad de México y Tlaxcala, mientras que para el periodo 2008-2018 ocurría algo similar en la Ciudad de México, Morelos, Estado de México y Puebla. Este comportamiento contrarrestó el de los sectores más dinámicos, de tal forma que en el agregado, el efecto diferencial fue negativo; es decir, estos dos últimos estados son poco competitivos.

Entre los estados que presentan mayor competitividad en relación con el resto está Guanajuato, que en ambos periodos tuvo alto dinamismo en los subsectores de curtido, acabado y fabricación de productos de cuero y piel, y en el de productos derivados del petróleo y del carbón. Este estado goza de condiciones de accesibilidad a los mercados o a los recursos productivos en comparación con otros estados.

Por su parte, para el efecto total, en el periodo 1998-2008 la Ciudad de México y Tlaxcala presentaron un efecto negativo, lo que implica que estos estados perdieron puestos de trabajo en comparación con el total regional. En ese sentido, podrían ser catalogados como "perdedores". En particular, en la Ciudad de México se perdieron 107,341 empleos y 90 en Tlaxcala; esto se debe al reordenamiento territorial a raíz de los cambios sufridos en la industria manufacturera, en donde los estados fronterizos del norte presentan múltiples ventajas (costos competitivos de transporte de insumos, bienes y servicios para la exportación o la importación) bajo el nuevo modelo de producción flexible y el contexto de una economía abierta al mercado mundial. Con ello, la participación de regiones tradicionalmente importantes por su actividad manufacturera, como la región centro del país, vieron disminuida signi- 
ficativamente su preponderancia a nivel nacional (Vieyra, 2000; Dávila, 2004; Maldonado, 2009).

Por su lado, para 2008-2018 la pérdida de empleos fue de 169,072 en la Ciudad de México; 695 en Hidalgo; 55,138 en el Estado de México; 2,875 en Morelos y 3,175 en Puebla. Estas pérdidas se deben, entre otros factores, al comportamiento de cada uno de los subsectores en cada estado y sus encadenamientos productivos con la industria de Estados Unidos, dada su alta integración con este país. En este sentido, la Pos Gran Recesión que se vivió durante estos años culminó en la destrucción de fuentes de empleo.

En cambio, los estados "ganadores" para el periodo 1998-2008 fueron Guanajuato, Hidalgo, Estado de México, Morelos, Puebla y Querétaro, los cuales presentaron un efecto total positivo. Esto indica que hubo un crecimiento en el empleo estatal, mayor que el crecimiento regional. Para el segundo periodo, los estados "ganadores" fueron Tlaxcala, con 3,913 nuevos empleos, Guanajuato con 152,241 y Querétaro con 74,804. Específicamente, en Querétaro y Guanajuato puede ser resultado de la implementación de medidas de política industrial que han permitido la innovación tecnológica, el mejoramiento de la infraestructura física, la capacitación profesional, el desarrollo territorial (renovación y ordenamiento), el apoyo a las pequeñas y medianas empresas, así como la condonación y/o reducción de contribuciones estatales, con exitosos resultados, apesar de la pérdida de empleos en la mayor parte de los estados analizados por los efectos posteriores a la Gran Recesión.

Asimismo, en 2008-2018 en la región tipo I (ganadora) se encuentran Guanajuato y Querétaro, los cuales están creciendo en el volumen de empleo por estar especializados en sectores dinámicos y de rápido crecimiento. El gran "ganador" fue Guanajuato, ya que permaneció en este tipo de región por 20 años, durante los que mantuvo su especialización, pero con fuerte liderazgo en el mercado interno en los subsectores del curtido, acabado y fabricación de productos de cuero y piel, y en productos derivados del petróleo y del carbón, además de que en los últimos años se empezó a especializar en la producción de aparatos eléctricos. Respecto a Querétaro, su especialización se ha dado a lo largo de dos lustros, en orden de importancia en: ${ }^{10}$ fabricación de acce-

10 La especialización de los subsectores se menciona en orden de importancia, dados sus valores del índice. 
sorios, aparatos eléctricos, maquinaria y equipo, equipo de transporte, madera, bebidas y tabaco, y papel. Estos estados son exportadores de los productos en los cuales se especializa. Salieron de este grupo el Estado de México y Morelos. El primero perdió su especialización en equipo de transporte, en la producción de minerales no metálicos y la industria química; mientras que el segundo estado la perdió en equipo de transporte, maquinaria y equipo, y productos de minerales no metálicos; es decir, en subsectores que generan grandes volúmenes de empleo.

Se aprecia que para 2008-2018 el Estado de México y Puebla se ubicaban en la región II-B (perdedora), lo que indica que existe la posibilidad de superar el rezago estatal por la vía de un aumento de la demanda nacional. El primero, a pesar de perder su especialización en algunos subsectores, la ha mantenido en el subsector de las metálicas básicas; plástico y hule; papel, productos metálicos; maquinaria y equipo; fabricación de colchones; insumos textiles; productos textiles; alimentos, y otras industrias; mientras que el segundo lo ha hecho en prendas de vestir, insumos textiles, productos de minerales no metálicos, productos textiles, equipo de transporte y madera. Por su parte, Puebla perdió importancia en las industrias metálicas básicas y en la fabricación de colchones y persianas.

Aunado a ello, Tlaxcala se clasificó en el último periodo como región III-A (ganadora); no obstante, a pesar de que creció más que el promedio, requiere una reconversión por su especialización en sectores poco dinámicos, ya que se especializa en insumos, acabados y productos textiles, prendas de vestir, productos no metálicos. Mientras que Hidalgo, Puebla y Querétaro salieron de este grupo. Al respecto, cabe mencionar que Querétaro mejoró su posición y Puebla e Hidalgo siguieron como "perdedores".

Respecto a Hidalgo, en el último periodo se ubica en la región III-B (perdedora) al especializarse en sectores poco dinámicos y de bajo crecimiento; en productos derivados de petróleo y carbón, prendas de vestir, insumos textiles, productos de minerales no metálicos y madera. En este caso la implicación es que es necesaria una reconversión productiva.

Por último, en la región tipo IV (perdedora) se ubican la Ciudad de México y Morelos. La primera tiene una especialización más diversificada; se observa que su especialización de empleo para ambos periodos fue en el subsector de impresión e industrias conexas, indus- 
tria química, de las bebidas y el tabaco, derivados del petróleo; fabricación de muebles, papel, plástico y hule; productos metálicos y otras industrias; sólo perdió su especialización en la industria alimentaria por los efectos de la Gran Recesión. Por su parte, la especialización de empleo para el estado de Morelos fue en los subsectores de la industria alimentaria, madera, bebidas y tabaco, y química. Esta entidad sí perdió su participación de empleo en subsectores importantes, ya mencionados; además, los subsectores en los que se especializa presentaban factores industriales en desventaja, por lo que es necesario el desarrollo de industrias en crecimiento e infraestructura productiva y social más activas para sacarlos del atraso, como canalizar recursos hacia los subsectores manufactureros más dinámicos, promover la exportación de sus productos y apoyar la investigación científica y tecnológica, entre otras medidas (Rendón et al., 2019b).

\section{b) Análisis shift-share con modificación de estructuras}

La diferencia entre el efecto inverso y el efecto estructural refleja la importancia del cambio estructural, denominado efecto estructural modificado, como se estableció en la sección metodológica. En el Cuadro 4 se puede observar un efecto positivo en Hidalgo, Morelos, Puebla y Querétaro para el primer periodo de estudio, lo que revela que la estructura regional estaba evolucionando en el periodo final hacia sectores productivos con un mayor dinamismo que a nivel regional. Estos estados, a excepción de Hidalgo, tenían una especialización particular, pero comparten una especialización en equipo de transporte. Por su parte, los estados con efecto regional modificado positivo fueron Guanajuato, Hidalgo, Estado de México, Morelos, Puebla, Querétaro y Tlaxcala, los cuales pueden clasificarse como "ganadores". 
CUADRO 4

TIPOLOGÍA Y RESULTADOS CON EL ANÁLISIS DIFERENCIAL ESTRUCTURAL MODIFICADO

PERSONAL OCUPADO MANUFACTURERO. 1998-2018

\begin{tabular}{ccccccccc}
\hline & \multicolumn{7}{c}{ 1998-2008 } \\
\hline $\begin{array}{c}\text { Sector/ } \\
\text { Región }\end{array}$ & $\begin{array}{c}\text { Ciudad de } \\
\text { México }\end{array}$ & Guanajuato & Hidalgo & $\begin{array}{c}\text { Estado de } \\
\text { México }\end{array}$ & Morelos & Puebla & Querétaro & Tlaxcala \\
\hline EEj & 2,176 & 3,981 & $-5,522$ & 9,176 & 1,727 & $-6,314$ & $-7,422$ & 494 \\
\hline EEMj & $-4,902$ & $-1,082$ & 78 & $-3,945$ & 2,989 & 2,436 & 6,822 & -692 \\
\hline ERMj & $-104,616$ & 28,566 & 16,538 & 20,806 & 5,161 & 7,623 & 25,815 & 108 \\
\hline Tipo & 10 & 5 & 7 & 5 & 1 & 7 & 7 & 6 \\
\hline EEj & $-30,879$ & 1,790 & $-7,749$ & 11,033 & -341 & 10,235 & 20,658 & $-4,747$ \\
\hline EEMj & 6,829 & 27,133 & 833 & $-21,581$ & -504 & $-13,809$ & $-1,133$ & 3,937 \\
\hline ERMj & $-145,023$ & 123,318 & 6,221 & $-44,590$ & $-2,033$ & 399 & 55,280 & 4,724 \\
\hline Tipo & 12 & 1 & 8 & 10 & 2 & 6 & 5 & 7 \\
\hline
\end{tabular}

Fuente: elaborado con datos del INEGI (1999, 2009 y 2020).

En contraparte, el signo negativo del efecto regional modificado en la Ciudad de México indica que se caracteriza por una especialización creciente en sectores en retroceso, perteneciente a una región "perdedora". Asimismo, se observa que el efecto regional modificado también es negativo en este caso, lo que implica que la Ciudad de México tuvo un menor crecimiento de personal ocupado que la media regional debido a factores internos. Más aún, la combinación de un efecto regional modificado y un efecto estructural modificado negativos implica que revertir esta tendencia puede ser complicado.

En suma, en este primer periodo sólo la Ciudad de México se considera "perdedora". Los demás estados se consideran "ganadores" y el orden de importancia de acuerdo con su tipología se aprecia en el Mapa 1. 
MAPA 1

TIPOLOGÍA DE RESULTADOS DEL MÉTODO DIFERENCIAL-ESTRUCTURAL MODIFICADO

REGIÓN CENTRO DE MÉXICO

PERSONAL OCUPADO. 1998-2008

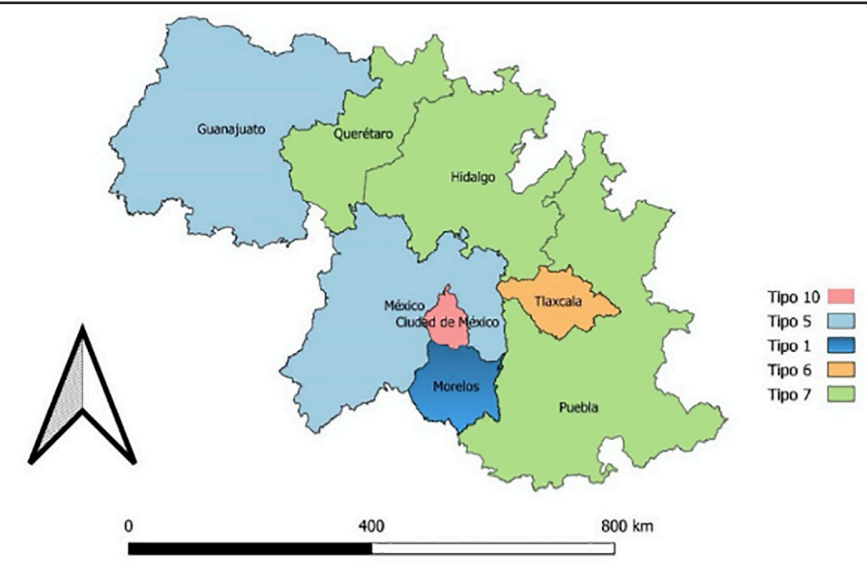

Fuente: elaboración propia.

Sin duda, el periodo Pos Gran Recesión tuvo efectos muy desfavorables en la mayoría de los estados de la región centro, como se observa en el Cuadro 4 y el Mapa 2. Los resultados son similares a la tipología del shift-share tradicional.

Por su parte, para el segundo periodo de estudio, dados los resultados del ERM, el gran ganador es Guanajuato, ubicado en la región tipo 1, lo que indica un crecimiento en el empleo, que continuará en el futuro. Es un estado con una dinámica manufacturera creciente, especialmente en el subsector de equipo de transporte y en el curtido, acabado de cuero y piel. Aquí se ha desarrollado una red de relaciones formales e informales entre empresas, intercambio de información en tecnología, mercado, maquinaria e insumos, que también ha dado lugar a una gran movilidad de trabajadores de una empresa a otra (Brown y Domínguez, 2013). A su vez, Querétaro y Tlaxcala mejoraron su posición en el volumen de empleo respecto al periodo anterior. En el primero, con efectos positivos en 13 de los 21 subsectores de la manufactura, sobresale la creación de empleos en el subsector del plástico $\mathrm{y}$ hule, $\mathrm{y}$ en el de equipo de transporte. Por su parte, el segundo tiene efectos positivos en 15 subsectores, principalmente en el de prendas de vestir. 
MAPA 2

TIPOLOGÍA DE RESULTADOS DEL MÉTODO DIFERENCIAL-ESTRUCTURAL MODIFICADO

REGIÓN CENTRO DE MÉXICO

PERSONAL OCUPADO. 2008-2018

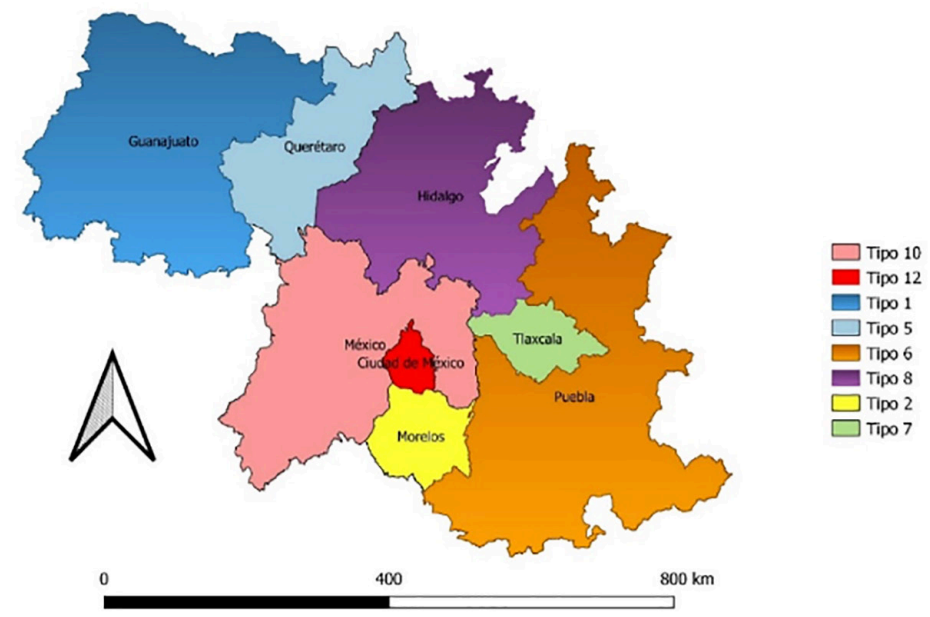

Fuente: elaboración propia.

Los estados con efectos adversos en este periodo fueron Morelos y Estado de México, los cuales han tenido una participación decreciente en el empleo regional. Ambos han perdido empleos en el subsector de equipo de transporte. ${ }^{11}$ No obstante, la situación se puede revertir en el futuro debido al cambio favorable en la mezcla industrial que han alcanzado. Por su parte, los estados que empeoraron respecto al periodo anterior fueron Hidalgo y Puebla, lo que indica que aún no sufren de la disminución de empleo en el total de los subsectores. Un análisis más detallado sugiere que el subsector más afectado ha sido el de equipo de transporte, sobre todo en Puebla. Es probable que este efecto alcance a

11 Como resultado de la crisis de las tres grandes empresas automotrices de EUA y de la trasmisión de la recesión de ese país a la economía mexicana, derivado del alto grado de sincronización del ciclo económico entre ambas economías, la producción de automóviles de la economía mexicana se ha visto negativamente impactada tanto en su mercado externo (en EUA) como en su mercado interno. No obstante, en el crecimiento experimentado por las exportaciones de automóviles en México en la década de los noventa se destaca que a partir del segundo trimestre de 2007, las importaciones de automóviles de México y Canadá iniciaron una tendencia decreciente que continuó a lo largo de 2008 y se ha profundizado en 2009 (Mendoza-Cota, 2011). 
los demás subsectores en el futuro dada la naturaleza de sus encadenamientos productivos.

Finalmente, la Ciudad de México se ubica en una región tipo 12, en peor situación que el periodo anterior, dada la disminución de sus puestos de trabajo. Pero antes de extraer conclusiones engañosas, se debe destacar que durante las últimas décadas se ha consolidado como "polo dominante de servicios", especialmente de los llamados servicios superiores, como las finanzas y publicidad, pero también de una gran diversidad de servicios más técnicos. De esta manera, la capital del país se ha mantenido como un punto de expulsión industrial hacia las aglomeraciones vecinas, como Puebla y Toluca, entre otras ciudades (Angoa et al., 2009).

\section{CONCLUSIONES}

En este trabajo se parte de que el crecimiento económico es mayor en algunos sectores productivos y en algunas regiones, lo que se confirma con la aplicación de la técnica shift- share tradicional y con modificación de estructuras al análisis de los subsectores de la manufactura de los estados del centro de México (Guanajuato, Querétaro, Estado de México, Hidalgo, Ciudad de México, Morelos, Tlaxcala y Puebla) durante los periodos 1998-2008 y 2008-2018.

Como se argumentó a lo largo del estudio, la especialización juega un papel importante en el resultado de estados "ganadores" y "perdedores" de empleo. Con ambas técnicas se observan consecuencias parecidas: destrucción de fuentes de empleo en el marco de una economía abierta y de mercado como consecuencia de la relocalización de las actividades productivas manufactureras hacia los estados del norte de México, por tener una mejor ubicación respecto al mercado estadounidense y formas de organización más flexibles, entre otras ventajas, así como por los efectos nocivos de la Gran Recesión, dada la alta integración con la economía de Estados Unidos de América.

Los resultados muestran que durante estos 20 años de estudio el subsector más afectado ha sido el de equipo de transporte, el cual había sido el más representativo en términos de generación de valor agregado y empleo, por no hablar de las exportaciones. De los ocho estados analizados, seis perdieron puestos de empleos y sólo Guanajuato y Tlaxcala se vieron favorecidos. Ambos se han especializado en subsectores diná- 
micos, que han adoptado estrategias para conformar plantas productivas relativamente integradas, con exportación de cantidades significativas de su producción y volúmenes importantes de inversión foránea. En particular, Guanajuato es considerado el gran "ganador" durante este periodo, debido a que ha sido capaz de consolidar las fortalezas competitivas de sus empresas de calzado, lo que le ha permitido profundizar en su especialización productiva basada en la formación de una cultura industrial original. En este caso se observan ventajas locales que favorecen la producción y la generación de empleo.

En general, el análisis shift-share con modificación de estructuras sugiere que Guanajuato, Hidalgo, Puebla, Querétaro y Tlaxcala se orientan a sectores más productivos en el segundo periodo de estudio.

Por su parte, la Gran Recesión parece haber afectado más a la Ciudad de México, la cual puede considerarse como la gran "perdedora" como resultado de su especialización en subsectores poco competitivos y dinámicos, con un volumen de empleo que ha crecido menos que el promedio regional. No obstante, debe tenerse en cuenta que durante las últimas décadas se ha ido desconcentrando deliberadamente su actividad industrial hacia los estados vecinos, lo que le ha permitido consolidarse, simultáneamente, como el polo dominante de servicios del alto valor agregado, no sólo de la región centro, sino del país.

En síntesis, el análisis presentado destaca la importancia que mantiene la región centro de México en el concierto nacional e identifica los subsectores manufactureros que explican el éxito de los estados "ganadores" y el retraso de los "perdedores" en la generación de empleo. En ese sentido, sienta las bases para el desarrollo de estudios posteriores que busquen tanto especificar a detalle las actividades que puedan impulsar la manufactura en los diferentes estados como diseñar estrategias de política pública para potenciar su desarrollo.

\section{Bibliografía}

Angoa, I., S. Pérez y M. Polése (2009), "Los tres Méxicos: análisis de la distribución espacial del empleo en la industria y los servicios superiores, por tamaño urbano y por región", Revista Latinoamericana de Estudios Urbano Regionales, 104 (35), pp. 121-144.

Banco de México (2018), Reporte sobre las Economías Regionales, Julio-Septiembre, Banxico. <www.banxico.org.mx> [13 de diciembre de 2019]. 
Barff, R. y P. Knight (1998), "Dynamic Shift-share analysis”, Growth and Change, 19 (2), pp. 1-10.

Barrios, F. (2008), "La influencia de la especialización productiva y regional en el comportamiento de las exportaciones colombianas del siglo XIX: un análisis con la metodología Shift Share", Panorama Económico, 16 (8), pp. 91-117.

Blair, J. (1995), Local Economic Development, Thousand Oaks, Sage Publications, EE.UU.

Boisier, S. (1980), "Técnicas de análisis regional con información limitada", Cuadernos del ILPES, 27, CEPAL-ILPES, Santiago de Chile.

Bonet, J. (1999), "El crecimiento regional en Colombia 1980-1996: una aproximación con el método shift and share", Documento de trabajo 10, Centro de Estudios Económicos Regionales, Banco de la República, Cartagena de Indias, Colombia, <www.banrep.gov.co/docum/economreg4.htm>.

Brown, F. y L. Domínguez (2013), "¿Tiene la industria aeronáutica mexicana las condiciones para integrarse a la cadena de valor internacional de alto valor agregado?", en Casalet M. (2013), La industria aeroespacial: Complejidad productiva e institucional, pp. 135-162. México: Flacso México.

Cañedo, Y., J. Lapa, N. Linares e I. Rodríguez (2012), “Breve revisión de las políticas regionales de fomento industrial en la Zona Metropolitana del Valle de México", en Issac-Egurrola, J. y Quintana, L. (coords.), La Industria en la Zona Metropolitana del Valle de México, vol. 2, Plaza y Valdés Editores, México, pp. 139-154.

Cárdenas, E. (1996), La política económica en México, 1950-1994, México, FCE.

Chavarría, J., L. Domínguez y F. Brown (2019), "La política gubernamental en la industria aeronáutica: un análisis comparativo de México, Brasil y España", Perfiles Latinoamericanos, 28(55), pp. 253-275.

Dávila, A. (2004), "México: concentración y localización del empleo manufacturero, 1980-1998”, Economía Mexicana. Nueva Época, XIII (2), pp. 209254.

Díaz, M., P. Mejía, A. Erquizio y R. Ramírez (2015), "Recesión en los estados de México en 2008-2009: magnitud y causas", Revista Contaduría y Administración, Universidad Nacional Autónoma de México, 60 (S2), pp. 147-168

Dunn, E. (1960), "A statistical and analytical technique for regional analysis", Paper and Proceedings of the Regional Science Association, 6 (1), pp. 97109.

Erquizio, A. y R. Ramírez (2017), “Crecimiento económico en México y manufactura global", Estudios Regionales en Economía, Población y Desarrollo, 40, pp. 3-30.

Esteban, J. (2000), "Regional convergence in Europe and the industry mix: a shift-share analysis", Regional Science and Urban Economics, 30 (3), pp. 353-364. 
Fuentes, A. (2003), “Apertura comercial y divergencia económica regional en México", Comercio Exterior, 53(10), pp. 970-978.

Fotopoulos, G., D. Kallioras y G. Petrakos (2009), "Spatial variations of Greek manufacturing employment growth: the effects of specialization and international trade", 89 (1), pp. 109-133.

Hanham R. y S. Banasick (2000), "Shift-Share Analysis and Changes in Japanese Manufacturing Employment”, Growth and Change, 31 (1), pp. 108-123.

Herath, J., P. Schaeffer y T. Gebremedhin (2013), "Employment change in LDs of west Virginia: a dynamic spatial shift-share analysis", American Journal of Rural Development, 1 (5), Science and Education publishing, pp. 99-105.

Hewings G. (1976), "On the accuracy of alternative models for stepping-down multi-county employment projections to counties", Economic Geography, 52, pp. 206-217.

Houston, D. (1967), "The Shift and Share Analysis of Regional Growth: A Critique", Southern Economic Journal, 33 (4), pp. 577-581.

Iacovone, L., F. Rauch y L. A. Winters (2013), “Trade as an engine of creative destruction: Mexican experience with Chinese competition", Journal of International Economics, 89, pp. 379-392.

Instituto Nacional de Estadística y Geografía (INEGI) (2020), Censos Económicos. $<$ www. inegi.org.mx $>$ [17 de julio de 2020].

Issac-Egurrola, J. y L. Quintana (2012), "La Zona Industrial de desarrollo del Valle de México”, en Issac-Egurrola, J. y Quintana, L. (coords.), La Industria en la Zona Metropolitana del Valle de México, serie Análisis Regional, 2, Plaza y Valdés Editores, pp.15 -23 y 215-325.

Klaassen, H. y H. Paelinck (1972), “Asymmetry in Shift-and Share Analysis,. Regional and Urban Economics, 3 (2), pp. 256-261.

Knudsen, D. (2000), "Shift-share analysis: further examination of models for the description of economic change", Socio-Economic Planning Sciences, 34 (3), pp. 177-198.

Knudsen, D. y R. Barff (1991), "Shift-Share Analysis as a Linear Model”, Environment and Planning A, 23, pp. 421-431.

Lira, L. y B. Quiroga (2003), “Técnicas de análisis regional”, Series Manuales, 30. ILPES-CEPAL.

Lira, L. y B. Quiroga (2009), "Técnicas de análisis regional”, Series Manuales, 59. ILPES-CEPAL.

Maldonado, A. (2009), "Parques industriales de México", Comercio Exterior, 59 (1), pp. 60-76.

Márquez, M., J. Ramajo y G. Hewings (2009), "Incorporating Sectoral Structure into Shift-Share Analysis", Growth and Change, A Journal of Urban and Regional Policy, 40 (4), pp. 594-618.

Mayor M. y A. López (2009), “Análisis espacial shift-share versus filtrado espacial: una aplicación a los datos laborales españoles”, en G. Arbia y Baltagi BH (eds.), Econometría espacial. Estudios de Economía Empírica, Physica-Verlag HD. 
Mejía, P. (2020), “Expansiones y recesiones en México, 1980-2019”, CICE-FEUAEMex, [manuscrito].

Mejía, P. (2011), "Sincronización nacional e internacional de la manufactura de los estados de México", en P. Mejía Reyes y M. E. Morales Fajardo (coords.), Integración y recesión económica en el binomio México-Estados Unidos, UAEMex, Toluca, pp. 211-240.

Mejía, P. y J. Campos (2011), "Are the mexican states and the United States business cycles synchronized? Evidence from the manufacturing production", Economía Mexicana. Nueva Época, XX (1), pp. 79-112.

Mejía, P. y V. Torres (2014), Efectos de las reformas estructurales en las fluctuaciones cíclicas y el crecimiento económico en México, UAEM / EÓN, México.

Mendez, O. (2015), "The effect of Chinese import competition on Mexican local labor markets", The North American Journal of Economics and Finance, 34, pp. 364-380.

Mendoza-Cota, J. (2011), “La crisis de la industria automotriz en México en el marco de la integración económica con Estados Unidos", Economía UNAM, 8 (22), pp. 55-73.

Mendoza, J. (2003), "Especialización manufacturera y aglomeración urbana en las grandes ciudades de México", Economía, Sociedad y Territorio, IV (13), pp. 95-126.

Mendoza, M. (2012), “Análisis macroeconómico de la industria manufacturera de la zona metropolitana del Valle de México (ZMVM): 1990-2010”, en J. Issac-Egurrola y L. Quintana (coords.), La Industria en la Zona Metropolitana del Valle de México, serie Análisis Regional, 2, Plaza y Valdés Editores, pp. 183-198.

Mitchell, W., J. Myers y J. Juniper (2005), "Extending Shift-share Analysis to Account for Spatial Effects: A Study Using Australian Census Data", working paper 19 (5), Centre of Full Employment and Equity, Callaghan, Australia.

Moreno-Brid, J. y J. Ros-Bosch (2010), Desarrollo y crecimiento en la economía mexicana. Una perspectiva histórica, FCE, México.

Nazara, S. y G. Hewings (2004), "Spatial Structure and Taxonomy of Decomposition in Shift-Share Analysis", Growth and change. Journal of urban and regional policy, 35 (4), pp. 476-490.

Ocegueda, J., R. Castillo y R. Varela (2009), "Crecimiento regional en México: Especialización y sectores clave", Problemas del Desarrollo, 40 (159), pp. 61-84

OCDE (2010), OECD Factbook 2010: Economic, Environmental and Social Statistics, < http://dx.doi.org/10.1787/ factbook-2010-en $>$ [23 de febrero de 2020].

Ramírez, R. (2012), "Evolución de los centros y subcentros industriales de la Ciudad de México 1994-2004”, en Issac-Egurrola, J. y Quintana, L. (coords.), La industria en la Zona Metropolitana del Valle de México, serie Análisis Regional, 2, Plaza y Valdés Editores, México, pp. 59-76. 
Rendon L., Roldan A. y P. Mejía (2019a), "Shift-share espacial del empleo manufacturero municipal. Zonas Metropolitanas: Valle de México y Toluca, 2008- 2013”, Economía, Sociedad y Territorio, 19 (59), pp. 1213-1242.

Rendón L., P. Mejía y R. Vergara (2019b), “Distribución espacial y especialización del empleo manufacturero de los municipios del centro de México, 1998-2013”, Revista Equilibrio Económico: Revista de Economía, Política y Sociedad, 15 (47), pp. 35-60.

Rodríguez, O. (2010), "La distribución territorial de la acumulación industrial metropolitana y sus efectos en los mercados de trabajo en la ZMCM 19852004”, Cuadernos de investigación, cuarta época, 59, Universidad Autónoma del Estado de México, Toluca.

Ros, J. (2015), ¿Cómo salir de la trampa del lento crecimiento y alta desigualdad, Colegio de México / UNAM, México.

Ros, J. (2014), Productividad y crecimiento en América Latina: ¿ por qué la productividad crece más en unas economias que en otras? CEPAL, México. $\quad<$ https://repositorio.cepal.org/bitstream/handle/11362/36770/1/LCMEXL1145s_es.pdf> [14 de marzo de 2020].

Silva, Iván (2003), "Disparidades, competitividad territorial y desarrollo local y regional en América Latina”, Serie Gestión pública, 33 (4), Comisión Económica para América Latina y el Caribe, Santiago de Chile, Chile. $<$ https:// repositorio.cepal.org/handle/11362/7286> [14 de febrero de 2018].

Sirakaya, E., H. Choi y T. Var (2002), "Shift-Share Analysis in Tourism: Examination of Tourism Employment Change in a Region", Journal of travel research, 8 (3), pp. 303-324.

Stilwell, F. (1969), "Regional Growth and Structural Adaptation", Urban Studies, 6 (2), pp. 162-178

Torres, F., R. Rózga, A. García y J. Delgadillo (2009), Técnicas de análisis regional. Desarrollo y aplicaciones, Trillas, México.

Vieyra, J. (2000), "Reconversión industrial, gran empresa y efectos territoriales. El caso del sector automotriz en México", EURE, 26 (77), pp. 25-47. 


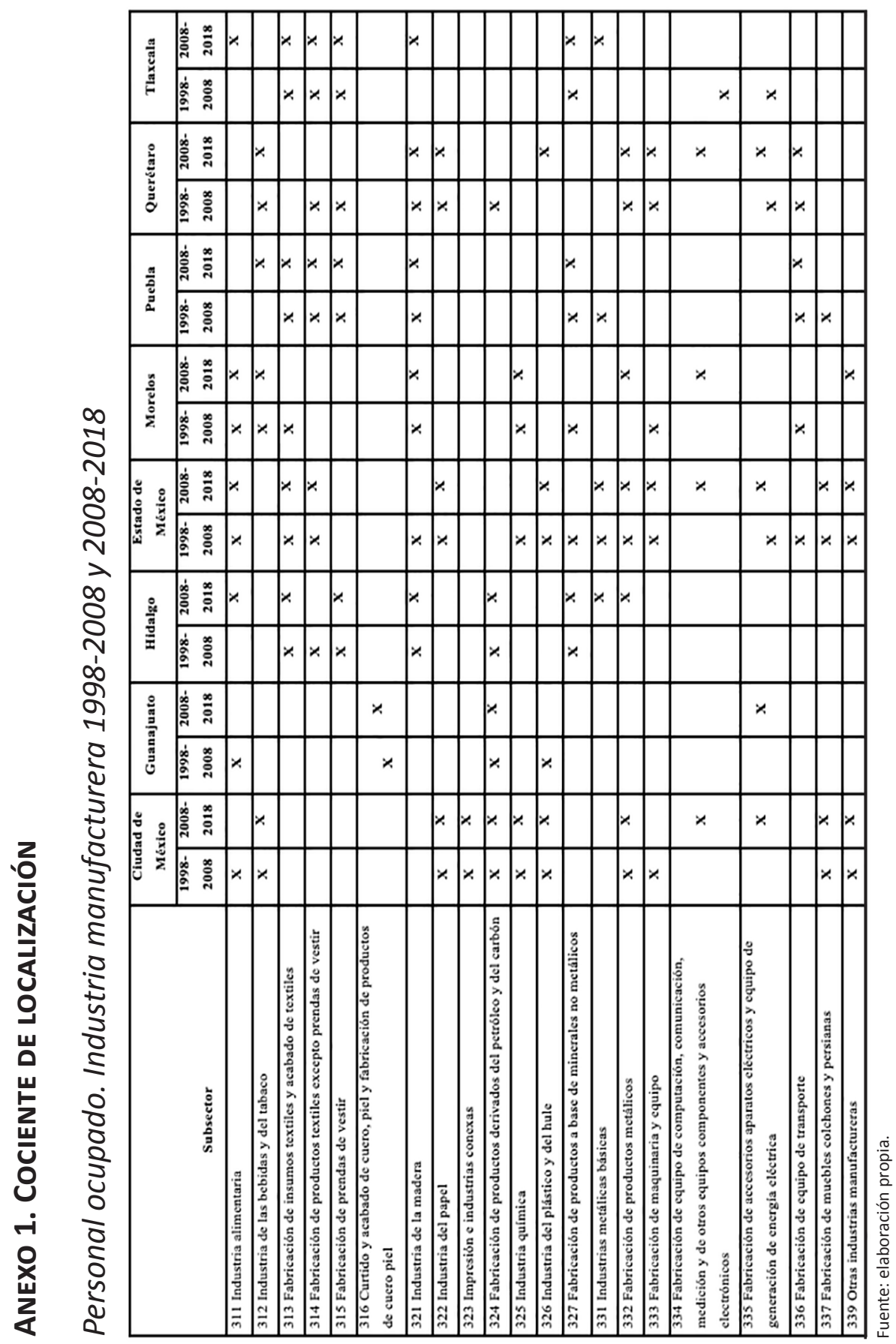

